\title{
The locus of spatial attention during the temporal integration of visual memories and visual percepts
}

\author{
JAMES R. BROCKMOLE, DAVID E. IRWIN, and RANXIAO FRANCES WANG \\ University of Illinois at Urbana-Champaign, Champaign, Illinois
}

\begin{abstract}
Temporal integration is a process by which two serially presented visual stimuli are mentally integrated to form a composite representation. In the present research, we explored how spatial selective attention is used during the delay separating stimuli, in order to determine the contents of spatial working memory in this task. A two-task situation was created. On the primary task, two dot arrays were serially presented within a grid, leaving one space empty, which subjects identified. On the secondary task, instead of the second array, a discrimination probe was presented. Integration accuracy increased through delays of $1,500 \mathrm{msec}$, revealing an estimate of the time required to form an optimal memory trace for integration. Once the memory trace was formed (but not before), response time to the probe was faster if it was presented in a location previously occupied by a dot from Array 1 . This indicates that during the delay separating the arrays, the subjects assigned spatial attention to the locations occupied by the first array and actively maintained the leading array in working memory. Implications for theories of visual processing and memory are discussed.
\end{abstract}

Temporal integration refers to a scenario in which information obtained from temporally distinct visual presentations is successfully combined to form a unified representation. An example of a temporal integration task is depicted in Figure 1. In this task, two dot arrays are serially presented within an enclosed square grid. In the process of presenting the arrays, all of the spaces in the grid are filled, save one. The subjects' task is to identify this space. Accuracy depends on the ability to retain the information contained in the first array and combine it with information in the second array. Accuracy, therefore, is an indicator of how well the two arrays were integrated across the intervening delay.

Past research has established that stimulus onset asynchrony (SOA), or the time that elapses between the onset of each array, is critical to performance. When the SOA (determined either by the duration of the first array or by the interval intervening between the arrays) is less than $50 \mathrm{msec}$, accuracy is very high in the temporal integration task, because of a phenomenon called perceptual integration. Perceptual integration results when a brief visual stimulus elicits activation in the visual system that outlasts the physical presentation of that stimulus. This residual activation is called visible persistence and causes the perception of a stimulus to continue beyond its offset. If a sec-

\footnotetext{
Support for this research was provided by a National Science Foundation Graduate Research Fellowship to J.R.B., National Science Foundation Grant SBR 96-15988 to D.E.I., and a University of Illinois Institutional Research Board Grant to R.F.W. We thank John Hogben and two anonymous reviewers for their helpful comments on this research, as well as David Kaneshiro and Derek Larson for their help with data collection. Address all correspondence concerning this article to J. R. Brockmole, now at Department of Psychology, Michigan State University, 129 Psychology Research Building, East Lansing, MI 48824 (email: jim@ eyelab.msu.edu).
}

ond stimulus is presented before the visible persistence of a previous stimulus has dissipated, activation generated by both stimuli will be present in the visual system simultaneously. These concurrent patterns of activation enable subjects to "see" both stimuli as present at the same time, even though the stimuli did not physically overlap in time (see Di Lollo, 1980, Di Lollo \& Hogben, 1987, Di Lollo, Hogben, \& Dixon, 1994, Dixon \& Di Lollo, 1994, Hogben \& Di Lollo, 1974, and Loftus \& Irwin, 1998, for detailed discussions of perceptual integration).

Perceptual integration, however, can occur only with very short SOAs. Visible persistence is time-locked to the onset of the stimulus (Di Lollo, 1980) and degrades within 100-150 msec (Coltheart, 1980). As such, perceptual integration is also restricted to a similar temporal window. If SOA is increased above $50-80 \mathrm{msec}$, accuracy declines dramatically and reaches a floor level by 100-200 msec. At this point, integration is virtually eliminated, because the visible persistence of the first array no longer overlaps with the second array.

Recently, Brockmole, Wang, and Irwin (2002) examined whether other visual memories, in addition to visible persistence, might support temporal integration. They reasoned that if subjects are afforded enough time to form a durable memory representation of the first array prior to the presentation of the second array, integration might be possible between the active memory trace and the subsequent percept. Such an effect may not have been observed in past research on integration because the SOA separating the arrays has seldom been investigated beyond 250$300 \mathrm{msec}$, which may not be enough time for consolidation of information from the lead array into a useful memory representation. Therefore, to investigate a possible memorypercept integration process, Brockmole et. al. systemati- 

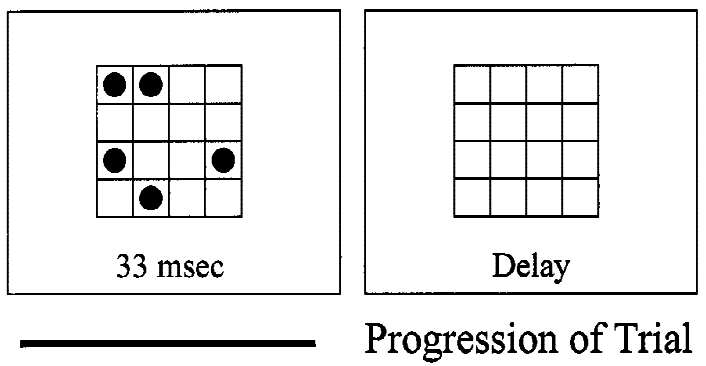

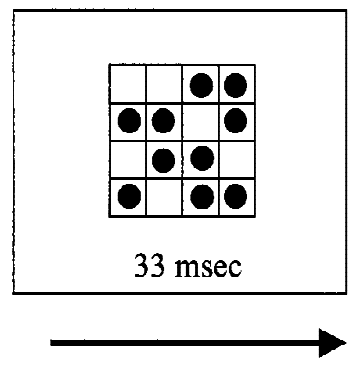

Figure 1. Schematic illustration of a temporal integration task. Following the presentation of two temporally distinct dot arrays, the subject identifies the grid space that was not filled during the trial.

cally increased the separation of the arrays through several thousand milliseconds. If, during that time, subjects could generate a useful memory representation of the first array that could be integrated with a later perceived visual stimulus, accuracy in the temporal integration task would improve from the floor levels observed at $100 \mathrm{msec}$. If, on the other hand, integration is not possible without visible persistence, no improvement would be possible.

Brockmole et al.'s (2002) major finding was that, as the separation between arrays increased above $100 \mathrm{msec}$, accuracy did indeed improve. Improvement evolved over a 1,500-msec period, after which it reached an asymptote that did not decline through separations of $5,000 \mathrm{msec}$. Strikingly, in some cases, the accuracy level after $1,500 \mathrm{msec}$ approached that achieved during perceptual integrationwhen the arrays are perceived simultaneously. An analysis of errors indicated that over $90 \%$ of the variance in accuracy was accounted for by a decrease in errors associated with the first array (i.e., erroneously selecting a grid position as the location of the missing dot that, in actuality, had been previously occupied by a dot from the first array). Brockmole et al. therefore argued that over a period of $1,500 \mathrm{msec}$, subjects generated a durable memory representation of the first array that could be integrated with the second array.

The purpose of the present experiment was to directly test the hypothesis that subjects in a temporal integration task can generate and actively maintain a memory representation of the lead array during the period separating the arrays, which they then integrate with the second array. The goal was to use a behavioral measure that directly reveals how subjects use spatial selective attention and spatial working memory during the temporal integration task. Specifically, the present experiment took advantage of a processing benefit that exists for stimuli that are presented within the spatial scope of selective attention (Posner, 1980). In fact, this processing benefit extends to stimuli presented in locations that are maintained in visual working memory (Awh, Jonides, \& Reuter-Lorenz, 1998). That is, stimuli are processed more quickly and more accurately if they are presented in spatial locations that are being attended or actively retained in memory.

The logic of the experiment reported here is based on Awh et al.'s (1998) finding that locations held in spatial working memory enjoy a visual-processing benefit because selective spatial attention is directed to those locations while they are rehearsed. In their paradigm, subjects judged whether two objects separated by $5,000 \mathrm{msec}$ were presented in the same location. Thus, the subjects had to maintain the location of the first object in spatial working memory. The critical manipulation was the insertion of an additional task into the design. During the 5,000-msec retention interval, a two-alternative forced-choice (2AFC) discrimination probe appeared on the screen. Response time (RT) to the probe was shorter when it appeared at the to-be-remembered location, indicating that spatial selective attention was directed toward the location maintained in spatial working memory.

The task employed by Awh et al. (1998) has an important similarity to the temporal integration task-namely, both tasks require subjects to maintain a memory for a spatial location previously occupied by an object and to use that memory in combination with a subsequent stimulus presentation to accomplish a goal. Therefore, the combination of the 2AFC task and the temporal integration task could enable one to directly determine the locus of spatial selective attention and, thus, spatial working memory in the temporal integration task.

In the present study, Brockmole et al.'s (2002) original procedure was modified to include a secondary $2 \mathrm{AFC}$ task similar to that used by Awh et al. (1998). Two trial types were created. The majority of the trials were integration trials. On these trials, the subjects were presented with two complementary dot arrays that filled all grid spaces but one. The subjects indicated which space was never filled, and accuracy was recorded. The remaining trials were probe discrimination trials. On these trials, instead of presenting the second array, the first array was followed by a $2 \mathrm{AFC}$ probe that appeared in a single grid space. The subjects indicated which of the two probes was presented, and RTs were recorded. The probes appeared either at a position occupied by the first array (on probe trials) or at a position left empty by the first array (off probe trials).

Integration trials were twice as common as probe discrimination trials, and the subjects did not know the nature of any particular trial beforehand. Accuracy in the primary task was stressed so that the subject would be en- 
couraged to maintain the first array in memory, in preparation for integrating it with the second array. Thus, spatial selective attention should be assigned to positions that were being maintained in visual working memory in preparation for the integration task. According to the hypothesis that subjects generate and maintain a memory representation of the first array during the delay separating the arrays, probes appearing at a location previously occupied by a dot from the first array (on probes) should be identified more quickly than when they appear elsewhere (off probes). However, because the memory representation forms over a period of 1,500 msec (Brockmole et al., 2002), any RT advantage for on probe trials may vary as a function of interstimulus interval (ISI). For example, prior to the complete generation of the memory trace, no RT advantage may be observed. To explore this issue, ISI was varied.

\section{METHOD}

\section{Subjects}

Fourteen University of Illinois undergraduates participated after providing informed consent. All the subjects were naive with respect to the experimental hypotheses and were compensated with partial course credit.

\section{Stimuli}

The stimuli were presented within an enclosed $4 \times 4$ square grid. The grid was composed of interconnected lines, drawn over the background so that the color of the grid spaces and the area surrounding the grid was the same. On integration trials, two unique dot arrays were presented. The first array contained 5 dots, and the second array contained 10 dots. Together, the arrays filled all but one square in the grid. On probe discrimination trials, an initial 5dot array was followed by a single discrimination probe. The probe was a $300^{\circ}$ arc (i.e., a circle with a $60^{\circ}$ gap). The arc opened either to the left or to the right.

The number of dots in each array was not equated, because of memory capacity limitation concerns. If the number of dots presented exceeds the number of dots that can be maintained in memory, some dot positions will be forgotten. In such a situation, if a probe appeared at a location occupied by a dot from the first array that had been forgotten, the trial would be scored as an on trial despite the fact that it was behaviorally equivalent to an off trial. Traditional measures of visual working memory capacity range from four to six items (Irwin, 1993; Irwin \& Andrews, 1996; Luck \& Vogel, 1997; Phillips, 1974; Pylyshyn \& Storm, 1988). Thus, presenting subjects with five to-be-remembered dots balanced the need to minimize the likelihood of the above situation while still creating a situation in which accuracy depended on the generation of a durable visual memory for the first array.

The subjects viewed the stimuli at a normal viewing distance from a computer screen (approximately $50 \mathrm{~cm}$ ). The total display subtended $38.1 \mathrm{~cm}$ horizontally (approximately $42^{\circ}$ of visual angle) and $27.9 \mathrm{~cm}\left(31^{\circ}\right)$ vertically. The square grid subtended $15.9 \mathrm{~cm}\left(18^{\circ}\right)$. Each square within the grid subtended $3.96 \mathrm{~cm}\left(4.5^{\circ}\right)$. Each dot presented in the array subtended $3.3 \mathrm{~cm}\left(3.8^{\circ}\right)$. The probe subtended $1.7 \mathrm{~cm}\left(2.0^{\circ}\right)$. The display background was light gray, the grid lines were light blue, the dots were black, and the probe was gray.

\section{Apparatus}

The stimuli were presented at a refresh rate of $60 \mathrm{~Hz}$ on a Gateway VX900 monitor. A Gateway E-4200 microcomputer controlled stimulus presentation and recorded responses. For both the integration and the probe detection tasks, responses were made using a mouse trackball.

\section{Design and Procedure}

Prior to the onset of each trial, an empty $4 \times 4$ square grid was presented in the center of the display. When ready to begin the trial, the subjects pressed the space bar. Immediately, an array of five dots was presented within the grid for $33 \mathrm{msec}$. This was followed by an ISI of $100,567,1,000,1,467,1,900,2,367$, or $2,800 \mathrm{msec}$, during which the empty grid remained on the screen. Following the ISI, one of two types of stimuli was presented, depending on the nature of the trial.

On integration trials, following the ISI, a second dot array consisting of 10 dots was presented for $33 \mathrm{msec}$. When combined with the first array, one position within the $4 \times 4$ grid was not filled. Under the constraint that no members of the two arrays should occupy the same spatial position, the dot patterns were randomly generated. Accordingly, the location of the missing dot was equally likely to occur in all the positions within the grid. The subjects were instructed to identify the position of the missing dot. They were specifically told that in order to do this, they were to remember the positions of the dots in Array 1 across the delay that separated the arrays and to combine this information with that contained in Array 2. Following the presentation of the second array, a cursor appeared on the screen. Using a mouse, the subjects moved the cursor to the position in the grid that they thought had been left empty during the trial and clicked both of the buttons. The subjects were told that they should respond as accurately as possible and that they were under no speed stress.

On probe discrimination trials, following the ISI, a single probe was presented in one grid space. Under the constraint that the probe should appear in a location occupied by a dot in the first array on one half of the trials, probe location was randomly selected. Accordingly, the location of the probe was equally likely to occur in all the positions within the grid. Each orientation of the probe (gap on left or right) occurred equally often. The subjects were instructed to indicate the orientation of the gap. The probe remained present on the screen until the subject made a response. To respond, the subjects clicked either the left or the right mouse button, depending on the location of the gap. On these trials, the subjects were told to respond as quickly and accurately as possible.

Trials were divided into blocks on the basis of ISI. The subjects were informed of the ISI duration prior to the start of each block. However, integration trials and probe detection trials were randomly intermixed, and the subjects were not informed which type a particular trial would be beforehand. The subjects completed 504 trials, divided into blocks of 12. Each ISI (seven) occurred equally often. However, integration and probe detection trials did not occur equally often: Two thirds of the trials (336 trials) were integration trials, and one third were probe detection trials (168 trials). Thus, integration trials occurred twice as often as probe detection trials. On probe trials, one half of the probes ( 84 trials) were presented in a grid space occupied by a dot from Array 1, and one half of the probes were presented elsewhere in the grid. For each subject, the blocks of trials were presented in a different random order.

Prior to beginning the experimental trials, the subjects completed two pure-task practice sessions to familiarize themselves with each task separately. In the first session, the subjects completed 50 integration trials. This session consisted of 10 trials each at ISIs of 0 , $100,1,000,2,000$, and 3,000 msec. In the second session, the subjects completed 40 probe discrimination trials. This session consisted of 8 trials each at ISIs of $0,567,1,000,1,467$, and 1,900 msec. During the practice sessions, the subjects were given feedback concerning the accuracy of their responses. However, during the experimental trials, no feedback was given. 


\section{RESULTS}

The results will be reported in two sections. First, accuracy and error rates for the integration task were assessed. The goal of these analyses was to establish that the present experiment replicated the original Brockmole et al. (2002) result that, as the ISI is increased beyond $100 \mathrm{msec}$, accuracy improves. Such replication is important because it establishes the appropriateness of analyzing RTs in the secondary probe discrimination task, since this analysis assumes that the subjects could engage in memory-percept integration. Second, RTs to the probe were analyzed as a function of probe location (on or off the first array) and ISI in order to determine the locus of selective visual attention and spatial working memory within the display during the course of a trial. An alpha level of .05 was adopted as the criterion for statistical reliability for all the analyses.

\section{Integration Task Accuracy and Error}

A response was classified as correct, an Array 1 error, or an Array 2 error. An Array 1 error was defined as an erroneously selected location that was previously occupied by a dot from Array 1. Likewise, an Array 2 error occurred when an erroneously selected location was previously occupied by a dot from Array 2. The rate of correct responses is a relative indicator of how well the arrays were integrated. The rates of Array 1 and Array 2 errors are relative indicators of how much information was remembered from each array, respectively: The more information that is remembered from a particular array, the less likely is an error associated with that array. Trends in accuracy and error are illustrated in the top panel of Figure 2 as a function of ISI. Accuracy, Array 1 error, and Array 2 error were measured in terms of the percentage of trials on which they occurred.

A one-way repeated measures analysis of variance with ISI as the independent factor demonstrated that accuracy varied as a function of ISI $\left[F(6,78)=19.7, M S_{\mathrm{e}}=75.7\right]$. Single degree of freedom polynomial tests indicated a reliable positive-slope linear trend $\left[F(1,13)=32.2, M S_{\mathrm{e}}=\right.$ $225.8]$ and quadratic trend $\left[F(1,13)=26.9, M S_{\mathrm{e}}=59.6\right]$ in the accuracy data. The linear trend indicates that accuracy increased as the ISI increased. The quadratic trend indicates that the rate of increase in accuracy was not constant across all the ISIs. That is, the relationship between accuracy and ISI was curvilinear. Post hoc contrasts showed that accuracy improved as the ISI increased through $1,467 \mathrm{msec}$ but did not differ when the ISI was greater than or equal to $1,467 \mathrm{msec}$.

In addition, the source of errors was assessed, with an emphasis on the strength of the relationship between the different types of errors and accuracy. As a percentage of total trials, Array 1 errors occurred, on average, on $46 \%$ of the trials and accounted for $76 \%$ of all the errors. The Pearson correlation between Array 1 errors and correct responses across ISIs was -.86 , revealing a very high level of dependence between accuracy and Array 1 error. This means that the improvement in accuracy was heavily due to the formation of a more complete memory representation of the first array. Array 2 errors, however, as a percentage of total trials, occurred, on average, on $14 \%$ of the trials and accounted for $24 \%$ of all the errors. The Pearson correlation between Array 2 errors and correct responses across ISIs was -.08 , revealing almost perfect independence between accuracy and Array 2 error. The pattern of results reported here closely parallels that observed by Brockmole et al. (2002).

\section{Probe Discrimination Response Time}

Prior to analyzing probe RT, trials were excluded if the probe discrimination was incorrect (2\% of the trials), or if RT was \pm 3 standard deviations of the mean RT for correct
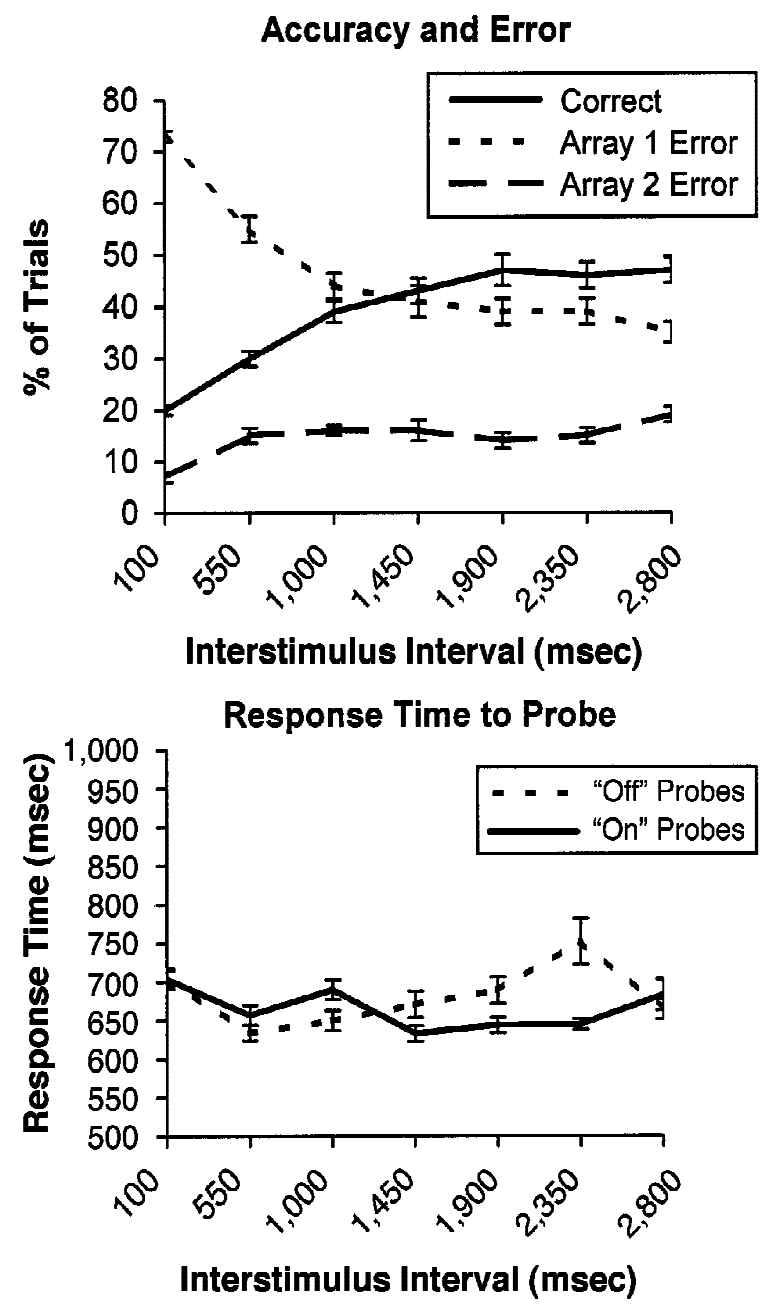

Figure 2. Experiment results. Top: mean correct responses, Array 1 errors, and Array 2 errors (with standard errors) for integration trials as the percentage of trials on which they occurred as a function of interstimulus interval (ISI). Bottom: mean response times (with standard errors) to the two-alternative forcedchoice probe on discrimination trials by probe type and interstimulus interval. 
trials, calculated on a subject-by-subject and cell-by-cell basis (1\% of the trials). Approximately $3 \%$ of the trials were excluded from the analyses by this trimming process. The remaining acceptable trials were submitted to a 2 (location of the probe: on or off first array) $\times 7$ (ISI) repeated measures analysis of variance. Mean RTs by probe location and ISI are illustrated in the bottom panel of Figure 2.

A main effect of ISI was observed $[F(6,78)=2.21$, $\left.M S_{\mathrm{e}}=6,074\right]$. This effect was driven primarily by a general decrease in RTs as ISIs increased through approximately $1,000 \mathrm{msec}$. This general trend is consistent with a foreperiod effect whereby RT decreases, to a point, as the time between a warning signal (in this case, Array 1) and a stimulus (in this case, the probe) increases (see Niemi \& Näätänen, 1981, for a review).

The analyses of critical importance, however, concerned differences in RTs based on the location of the probe. A marginal main effect of probe location was observed $[F(1,13)=2.94, p=.11]$. More important, a reliable interaction between probe location and ISI was present $\left[F(6,78)=2.38, M S_{\mathrm{e}}=7,734\right]$. Post hoc contrasts were conducted to explore the nature of this interaction. Analyses revealed that, for ISIs less than or equal to $1,000 \mathrm{msec}$, RTs for off probes $(M=663 \mathrm{msec})$ were, on average, $21 \mathrm{msec}$ shorter than those for $o n$ probes $[M=684 \mathrm{msec}$, $\left.F(4,13)=4.63, M S_{\mathrm{e}}=11,538\right]$. This pattern, however, reversed for ISIs greater than or equal to $1,467 \mathrm{msec}$, where RTs for on probes $(M=651 \mathrm{msec})$ were, on average, $43 \mathrm{msec}$ shorter than those for off probes $(M=694 \mathrm{msec})$. Note that this crossover occurs exactly when accuracy in the primary task reaches its maximum level.

\section{DISCUSSION}

As the ISI increased above $100 \mathrm{msec}$, accuracy on the integration task improved through approximately $1,500 \mathrm{msec}$, after which an asymptotic level was attained. RTs in the probe discrimination task also varied with ISI, and the temporal properties of this variation closely matched those associated with integration accuracy. Prior to the point at which accuracy reached asymptote $(1,467 \mathrm{msec})$, RTs to off probes were shorter than those to on probes (this effect may be due to forward masking when the arrayto-probe interval was short: Masking would be greater for on probes, and thus, RTs to on probes would be slowed relative to off probes). Critically, however, once integration accuracy reached asymptote - the point at which spatial attention is most focused on the aspects of the display remembered or rehearsed during the ISI-on probes were discriminated more quickly than off probes, indicating that, at this point during the delay, spatial attention held the locations of the first array in memory.

The coupling of accuracy improvement in the integration task and the development of the on probe advantage in the probe detection task supports the hypothesis that temporal integration is possible outside the temporal window of perceptual integration if subjects are afforded time to develop a representation of the first array. When asymp- totic performance on the integration task was observed, spatial attention and memory were assigned to spatial locations that corresponded to the positions of the previous stimulus. If integration was required prior to this point (i.e., the ISI between arrays was less than $1,467 \mathrm{msec}$ ), performance was suboptimal. The present results suggest that lower levels of performance in this time interval may be due to the inadequate focus of spatial attention or to a lessthan-ideal memory representation.

Although integration is possible between visual percepts separated by $1,000-5,000 \mathrm{msec}$, it need not necessarily occur. In fact, an interesting contrast can be made between the present evidence for memory-percept integration and another phenomenon known as visual marking, where active inhibition of items in prior visual displays takes place. In visual-marking experiments, subjects engage in a standard conjunction search task (e.g., find the blue $H$ target among red $H$ and blue $S$ distractors). In such tasks, RT to find the target increases as a function of the number of distractors in the display (Treisman \& Gelade, 1980). However, if one set of distractors (e.g., red $H \mathrm{~s}$ ) is presented 1,000 msec before the other set of items (e.g., the blue $S$ s and the blue $H$ ), RT to find the target is unaffected by the number of distractors in the first set. This result suggests that subjects are able to visually mark the first set of distractors as bogus items and search only through the items presented in the second set (Watson \& Humphreys, 1997). Critically, when a secondary probe detection task is inserted into this paradigm, the probes that appear at the location of a new item are detected more quickly than probes that appear at the location of an old item. This suggests that subjects actively inhibit the old locations (Watson \& Humphreys, 2000).

Thus, it seems that, when combining visual information across temporally disparate visual presentations, two different mechanisms can be recruited. In Watson and Humphreys's $(1997,2000)$ visual-marking experiments, the items in the lead display were actively inhibited, allowing for segregation of information. However, in the present memory-percept integration experiment, the items in the lead display were actively attended and maintained in memory, allowing for a summation of information across time. How each of these processes are employed during everyday perception of natural scenes and what perceptual or cognitive factors influence each of them are open and important avenues for future research.

\section{REFERENCES}

Awh, E., Jonides, J., \& Reuter-Lorenz, P. A. (1998). Rehearsal in spatial working memory. Journal of Experimental Psychology: Human Perception \& Performance, 24, 780-790.

Brockmole, J. R., Wang, R. F., \& Irwin, D. E. (2002). Temporal integration between visual images and visual percepts. Journal of Experimental Psychology: Human Perception \& Performance, 28, 315-334. Coltheart, M. (1980). Iconic memory and visible persistence. Perception \& Psychophysics, 27, 183-228.

Di Lollo, V. (1980). Temporal integration in visual memory. Journal of Experimental Psychology: General, 109, 75-97.

Di Lollo, V., \& Hogben, J. H. (1987). Suppression of visible persis- 
tence as a function of spatial separation between inducing stimuli. Perception \& Psychophysics, 41, 345-354.

Di Lollo, V., Hogben, J. H., \& Dixon, P. (1994). Temporal integration and segregation of brief visual stimuli: Patterns of correlation in time. Perception \& Psychophysics, 55, 373-386.

Dixon, P., \& Di Lollo, V. (1994). Beyond visible persistence: An alternative account of temporal integration and segregation in visual processing. Cognitive Psychology, 26, 33-63.

Hogben, J. H., \& Di Lollo, V. (1974). Perceptual integration and perceptual segregation of brief visual stimuli. Vision Research, 14, 10591069.

IRWIN, D. E. (1993). Memory for spatial position across saccadic eye movements. In G. d'Ydewalle \& J. Van Rensbergen (Eds.), Perception and cognition: Advances in eye-movement research (pp. 323332). Amsterdam: North-Holland.

IRWIN, D. E., \& ANDREWS, R. V. (1996). Integration and accumulation of information across saccadic eye movements. In T. Inui \& J. L. McClelland (Eds.), Attention and performance XVI: Information integration in perception and communication (pp. 125-155). Cambridge, MA: MIT Press.

LofTus, G. R, \& IRwin, D. E. (1998). On the relations among different measures of visible and informational persistence. Cognitive Psychology, 35, 135-199.
Luck, S. J., \& Vogel, E. K. (1997). The capacity of visual working memory for features and conjunctions. Nature, 390, 279-281.

NiEMI, P., \& NäÄTÄNEN, R. (1981). Foreperiod and simple reaction time. Psychological Bulletin, 89, 133-162.

Phillips, W. A. (1974). On the distinction between sensory storage and short-term visual memory. Perception \& Psychophysics, 16, 283-290.

Posner, M. I. (1980). Orienting of attention. Quarterly Journal of Experimental Psychology, 32, 3-25.

Pylyshyn, Z. W., \& STORM, R. W. (1988). Tracking multiple independent targets: Evidence for a parallel tracking mechanism. Spatial Vision, 3, 179-197.

Treisman, A., \& Gelade, G. (1980). A feature-integration theory of attention. Cognitive Psychology, 12, 97-136.

Watson, D. G., \& Humphreys, G. W. (1997). Visual marking: Prioritizing selection for new objects by top-down attentional inhibition. Psychological Review, 104, 90-122.

Watson, D. G., \& Humphreys, G. W. (2000). Visual marking: Evidence for inhibition using a probe-dot detection paradigm. Perception \& Psychophysics, 62, 471-481.

(Manuscript received January 28, 2002; accepted for publication April 24, 2002.)

\title{
Nominations for the Editorship of Behavior Research Methods, Instruments, \& Computers
}

Nominations are solicited for the editorship of BRMIC. The term of the present editor, Jonathan Vaughan, expires at the end of 2004. The new editor will begin an official 4- or 5-year term on January 1, 2005, and will begin to receive manuscripts early in January 2004. The Publications Committee of the Psychonomic Society expects to appoint the new editor by November 2003.

Nominations (including self-nominations) should be submitted by August 31, 2003, to:

\author{
C. R. Gallistel \\ Chair, BRMIC Search Committee \\ Department of Psychology, RUCCS \\ 152 Frelinghuysen Road \\ Piscataway, NJ 08854-8020 \\ galliste@ruccs1.ntgers.edu
}

